\title{
Necrosis cutánea por warfarina
}

\section{Warfarin-Induced skin necrosis}

\author{
Edison Muñoz, Óscar Jairo Valencia, Luis Guillermo Toro, \\ luis Alfonso Correa, Juan Carlos Wolff • Medellín (Colombia)
}

\section{Resumen}

La necrosis cutánea por warfarina aparece entre $0.01-0.1 \%$ de los pacientes, afecta más a mujeres con obesidad y antecedentes de enfermedad tromboembólica luego del quinto día de su inicio. Se debe a un imbalance transitorio de los sistemas anticoagulante y procoagulante, caracterizado por flictenas hemorrágicas en zonas con tejido graso. Reportamos el caso de una necrosis cutánea por warfarina con un desenlace fatal. (Acta Med Colomb 2012; 37: 138-141)

Palabras clave: necrosis, piel, warfarina, anticoagulantes, efectos adversos.

\begin{abstract}
Warfarin-induced skin necrosis appears between $0.01 \%$ to $0.1 \%$ of patients. It affects mostly women with obesity and a history of thromboembolic disease after the fifth day of its administration, and is caused by a transient imbalance of procoagulant and anticoagulant systems, characterized by hemorrhagic blisters in areas with fatty tissue. (Acta Med Colomb 2012; 37: 138-141)

We report the case of a warfarin-induced skin necrosis with fatal outcome.

Keywords: necrosis, skin, warfarin, anticoagulants, adverse effects.
\end{abstract}

Dr. Edison Muñoz Ortiz: Residente de Medicina Interna, Facultad de Medicina Universidad de Antioquia; Dr. Óscar Jairo Valencia Ocampo: Residente de Dermatología, Facultad de Medicina Universidad de Antioquia; Dr. Luis Guillermo Toro: Internista, Hospital Universitario San Vicente Fundación. Docente Facultad de Medicina Universidad de Antioquia; Dres. Luis Alfonso Correa López y Juan Carlos Wolff Idarraga: Dermatopatólogos, Docentes Facultad de Medicina Universidad de Antioquia. Medellín (Colombia).

Correspondencia: Dr. Edison Muñoz Ortiz. Medellín (Colombia).

E-mail: edisonhoy@yahoo.es

Recibido: 02/VIII/2011 Aceptado: 20/VIII/2012

\section{Caso clínico}

Se trata de una mujer de 86 años, con antecedentes patológicos de múltiples comorbilidades que incluían una insuficiencia cardiaca severa con clase funcional NYHA IV (New York Heart Association), diabetes mellitus tipo 2 e hipertensión arterial; había permanecido postrada en cama durante el último año y presentó trombosis venosa profunda de la vena femoral común izquierda un mes antes de su nueva consulta; desde entonces se encontraba en tratamiento con warfarina $5 \mathrm{mg} /$ día. Consultó por la presencia de ampollas en piel, con un cuadro de 15 días de evolución de lesiones tipo placas equimóticas que rápidamente progresaban a flictenas tensas de contenido hemorrágico localizadas en muslo derecho, con posterior aparición en pierna derecha. Ingresa a una institución de tercer nivel de la ciudad de Medellín, Colombia, en regulares condiciones generales, con signos de insuficiencia cardiaca. Al examen físico presentaba equimosis, edema, frialdad y disminución de la sensibilidad en cuarto y quinto dedos de pie izquierdo (Figura 1); una flictena tensa de contenido hemorrágico, de 25 por $12 \mathrm{~cm}$ en cara posterior de la pierna derecha (Figura 2), máculas hiperpigmentadas residuales y zonas de exulceración superficial en muslo derecho. Con las impresiones diagnósticas de una necrosis cutánea por warfarina e isquemia crítica por trombosis aguda versus

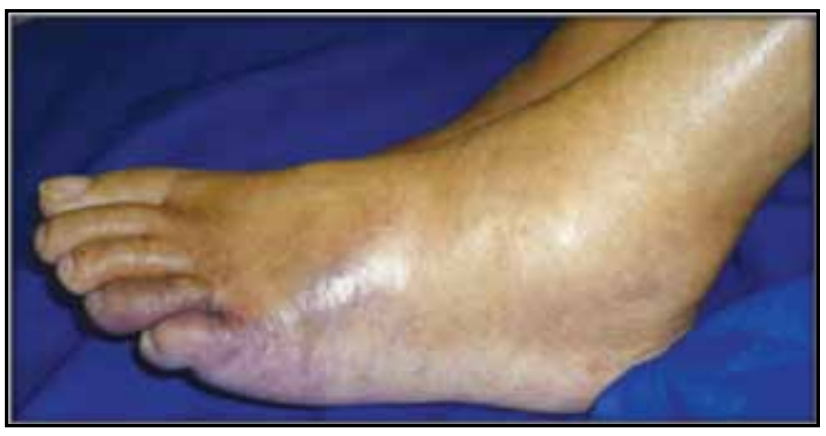

Figura 1. Equimosis, edema y mala perfusión en cuarto y quinto dedos de pie izquierdo.

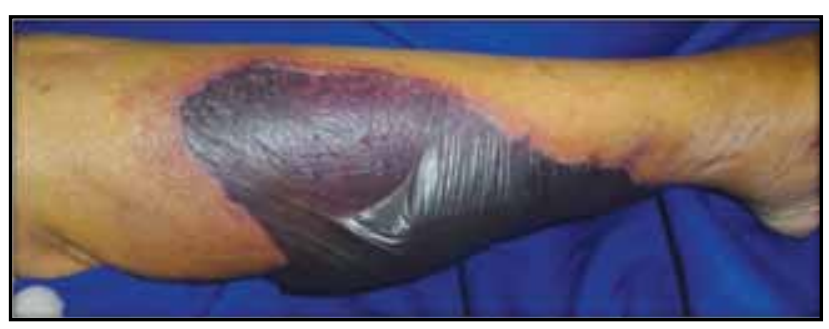

Figura 2. Flictena de contenido hemorrágico, de 25 por $12 \mathrm{~cm}$ en cara lateral y posterior de la pierna derecha. 
microembolismos de colesterol en dedos de pies, se tomaron dos biopsias de piel, la primera del pie izquierdo (parches equimóticos dorso del quinto dedo) y la segunda de la pierna derecha (ampolla hemorrágica). Para ambas se reportaron hallazgos histopatológicos similares, caracterizados por vasculopatía trombótica de pequeños y medianos vasos, sin infiltrado inflamatorio acompañante, ni signos de vasculitis (Figuras 3 y 4). Los exámenes paraclínicos mostraban unos tiempos de coagulación prolongados, hipoalbuminemia y anemia moderada; no se realizaron estudios de hipercoagulabilidad. Con base en los hallazgos clínicos soportados en la histopatología, se hizo un diagnóstico definitivo de necrosis cutánea por warfarina y trombosis aguda de dedos del pie izquierdo. Desde el primer día de hospitalización se suspendió la warfarina, y se inicio anticoagulación con heparina de bajo peso molecular (enoxaparina), además de tratamiento de soporte, al tercer día del ingreso la paciente fallece por enfermedad isquémica crónica.

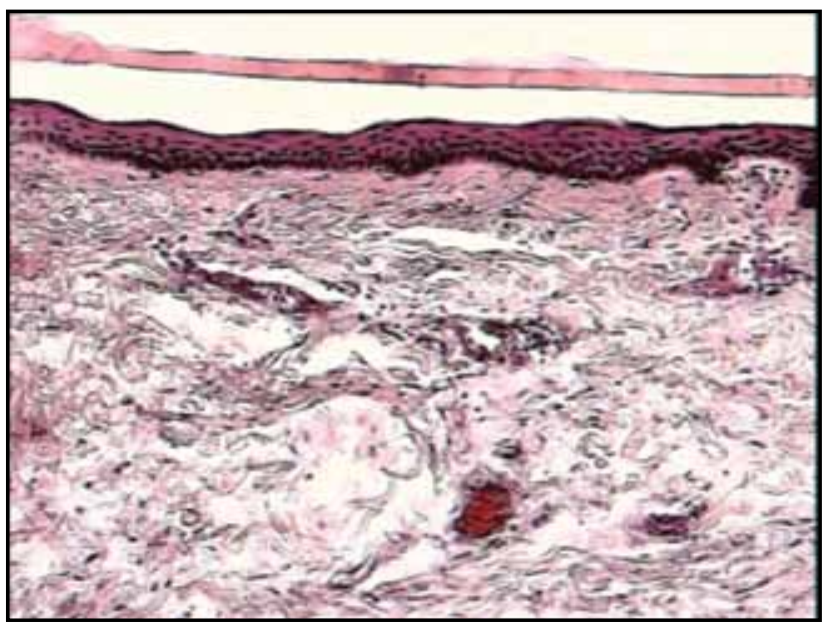

Figura 3. Microfotografía de una de las lesiones hemorrágicas de la paciente. Se observan pequeños vasos ocupados por trombos sin signos de vasculitis ni reacción inflamatoria. H-E X10.

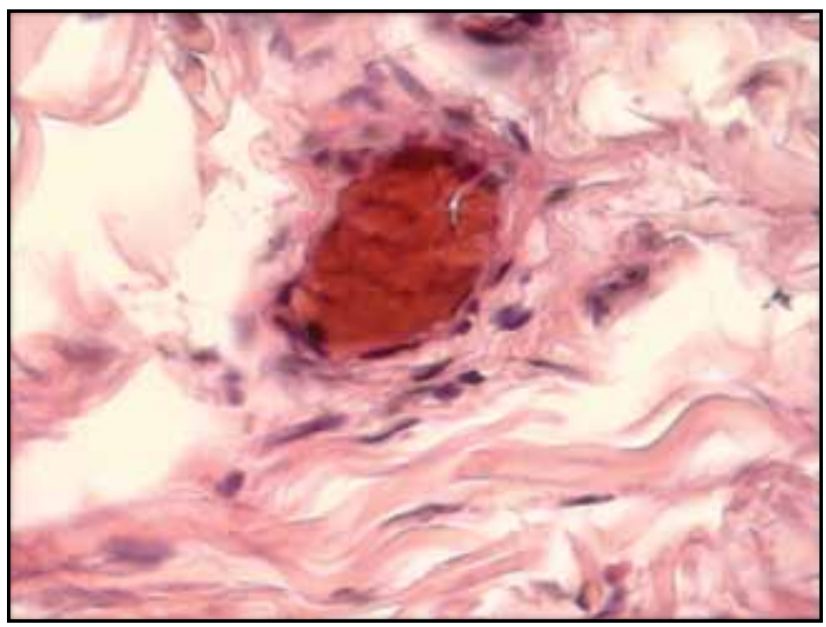

Figura 4. Microfotografía de una de las lesiones hemorrágicas de la paciente. Detalle de uno de los vasos trombosados. H-E X40.

\section{Discusión}

La warfarina es un anticoagulante oral, antagonista de la vitamina $\mathrm{K}$, la cual es una mezcla racémica de enantiómeros S y R (1). Su efecto anticoagulante esta mediado por la inhibición de los factores vitamina $\mathrm{K}$ dependientes de la gamma-carboxilación (II, VII, IX y X) (2), lo que da como resultado la síntesis de proteínas de la coagulación que pueden ser detectables de manera inmunológica, pero que carecen de actividad biológica. Pero además, la warfarina también inhibe las proteínas C y S dependientes de la gamma-carboxilación, éstas tienen propiedades anticoagulantes a través de la inactivación de dos cofactores de la coagulación, el factor V y VIII activado (1). Es por estos efectos competitivos que los cumarínicos como la warfarina crean una paradoja bioquímica, pues producen un efecto anticoagulante (por inhibición de los factores II, VII, IX y $\mathrm{X}$ ) y un efecto potencialmente trombogénico por alteración de la síntesis de anticoagulantes naturales (proteína C y S).

Múltiples efectos adversos se han asociado con el uso de la warfarina: sangrado, priapismo, hepatitis, alopecia, erupción pruriginosa macular y papular, síndrome del dedo azul (microembolización de colesterol) y necrosis cutánea (1).

\section{Necrosis cutánea por warfarina}

La necrosis inducida por warfarina es una rara pero bien descrita complicación de la terapia con cumarínicos, la cual se asocia con una alta morbilidad y mortalidad.

En 1943 se describieron los cambios necróticos en la piel de un paciente que tomaba warfarina, estos cambios fueron denominados "tromboflebitis migrans diseminada" (3). Sin embargo, para este momento los hallazgos en la piel no fueron relacionados con el consumo de warfarina, sólo fue hasta 1954 cuando Verhagen reportó 13 casos de necrosis cutánea inducida por warfarina (4).

Estas lesiones se presentan aproximadamente en el 0.01 a $0.1 \%$ de todos los pacientes que reciben warfarina sódica, con una predilección por el sexo femenino (70 - 90\%), siendo el paciente típico una mujer de mediana edad, obesa, con historia de trombosis venosa profunda o tromboembolia pulmonar, ya que éstos se relacionan más con la presencia de deficiencia de proteína $\mathrm{C}$ y $\mathrm{S}$ que aquellos pacientes que están anticoagulados por otros motivos, como por ejemplo por la presencia de una fibrilación auricular (5).

Está complicación del uso de warfarina no es común, de tal manera que revisando la literatura disponible se han reportado aproximadamente 300 casos de esta patología. Lo típico es que las lesiones se desarrollen durante los primeros días luego del inicio de la terapia con warfarina (usualmente alrededor del decimo día), a menudo asociada con la administración de dosis de carga (6). Sin embargo, también se encuentran en la literatura reportes de presentaciones tan tardías como a los tres años luego del inicio del cumarínico (7).

En estos casos la hipótesis es que en algún momento se pudo haber suspendido la anticoagulación, haber disminuido 
los niveles de manera transitoria o presentarse un estado procoagulante (sepsis, falla cardiaca, hepatopatía, por ejemplo), y una vez reiniciada la anticoagulación se desarrolla la necrosis. Esto es posible ya que la proteína $\mathrm{C}$, que es sintetizada por el hígado, tiene una vida media corta y su concentración en sangre se reduciría en aquellas patologías que afectaran el hígado antes que los factores procoagulantes (8). Incluso podría ocurrir por interacción con otros medicamentos que disminuyen los niveles séricos o aumentan el metabolismo de la warfarina, como ocurrió en el reporte de Cameron et al. (7), en donde la necrosis inducida por warfarina se relacionó con el uso de salicilatos (4 g/día) durante cuatro días previos al evento, posiblemente por desplazamiento de la warfarina ligada a la albúmina, resultando en un estado procoagulante transitorio.

El mecanismo fisiopatológico exacto de esta entidad permanece incierto, la trombosis microvascular, la hipersensibilidad a la warfarina y un efecto tóxico directo de ésta han sido postulados (8). Sin embargo, el mecanismo más probable pareciera ser un imbalance transitorio entre los sistemas anticoagulante-procoagulante, más específicamente relacionado con un rápido descenso en los niveles de proteína $\mathrm{C}$ durante la terapia inicial con warfarina.

Se postula que podría estar asociada con la deficiencia hereditaria de proteína $\mathrm{C}$. La resistencia a la proteína $\mathrm{C}$ activada se encuentra en 5\% de la población general, pero en la gran mayoría de los casos, la necrosis inducida por warfarina no se asocia a deficiencia de ésta. Sin embargo, los pacientes con deficiencia heterocigota tienen un riesgo mucho más alto (8) y parece que la terapia con proteína $\mathrm{C}$ no sólo detiene las lesiones, sino que también promueve su resolución (9).

El paciente primero presenta una erupción eritematosa mal delimitada que a menudo se asocia a edema de tejidos blandos y parestesias, posteriormente aparecen petequias, que en el lapso de horas progresan a equimosis y grandes ampollas de contenido hemorrágico, para terminar en una necrosis franca, que puede comprometer el espesor total de la piel, siendo la mayoría de los casos lesiones únicas, aunque hasta en un tercio de los casos se desarrollan lesiones múltiples. Estas lesiones se pueden desarrollar en cualquier parte del cuerpo, pero tienen predilección por áreas con alto contenido graso como son las mamas, glúteos, muslos, los brazos, las manos, los dedos, las piernas, pies, cara y abdomen y en los hombres se puede afectar la piel del pene (6). Hay pocos reportes de curación espontánea una vez se ha suspendido la warfarina, y no siempre recurren con la reintroducción de la misma.

Para el diagnóstico se requiere un alto grado de sospecha, para un rápido reconocimiento y manejo, que podría salvar la vida del paciente (4). Una vez se reconocen las lesiones, se debe suspender la warfarina, se inicia manejo con heparina no fraccionada o heparina de bajo peso molecular, para evitar formación de otros trombos, se aconseja el uso de vitamina $\mathrm{K}$ y plasma fresco congelado para una rápida reposición de los niveles de proteína C y S (6). También se puede utilizar concentrados de proteína $\mathrm{C}$ que como se había expresado previamente parece que detiene la progresión de las lesiones y favorece la curación, pero en muchas ocasiones su precio podría hacer esto prohibitivo (9). Más de 50\% de los pacientes requerirán manejo quirúrgico y desbridamiento extenso.

La prevención de la necrosis por warfarina se podría lograr evitando la dosis de carga con warfarina y con la coadministración de heparina no fraccionada o heparina de bajo peso molecular como terapia puente al menos durante los primeros cinco días. Por otro lado, el tamizaje para deficiencia de proteína $\mathrm{C}$ y $\mathrm{S}$ no es costo efectivo, ya que muchos pacientes con deficiencia documentada no desarrollan esta entidad. Se han intentado otras opciones de tratamientos como vasodilatadores o esteroides, pero éstos no alteran el curso de esta patología (8).

En el diagnóstico diferencial, se deben tener en cuenta varias patologías (Tabla 1) (10), dentro de las que se destacan trastornos de la coagulación como el síndrome antifosfolípido, el síndrome HIT (trombocitopenia inducida por heparinas) en la que se pueden presentar eventos trombóticos, la púrpura trombótica trombocitopénica, en la que aparecen fenómenos trombóticos y hemorrágicos acompañados de la presencia de trombocitopenia y esquistocitos en el extendido de sangre periférica, hallazgos que también se identifican en la coagulación intravascular diseminada (11); y algunos trastornos hematopoyéticos como los síndromes mieloproliferativos crónicos con manifestaciones trombóticas por hipercoagulabilidad secundaria a la neoplasia hematológica, o la hemoglobinuria paroxística nocturna donde además de la anemia hemolítica intravascular y otras citopenias, pueden presentarse eventos trombóticos (12).

\section{Conclusión}

Se presentó un caso representativo de una necrosis cutánea por warfarina asociada a una trombosis aguda de dedos de pie, complicación poco frecuente de alta morbimortalidad, un reconocimiento temprano y la correlación clínico-

Tabla 1. Diagnóstico diferencial de la necrosis cutánea por warfarina.

\begin{tabular}{|c|}
\hline Trombocitopenia inducida por heparina \\
\hline Púrpura trombótica trombocitopénica \\
\hline Coagulación intravascular diseminada \\
\hline Púrpura fulminans \\
\hline Síndromes mieloproliferativos crónicos \\
\hline Hemoglobinuria paroxística nocturna \\
\hline Fascitis necrotizante \\
\hline Calcifilaxis \\
\hline Crioglobulinemia \\
\hline Microembolias de colesterol \\
\hline Úlceras por decúbito \\
\hline Carcinoma de mama inflamatorio \\
\hline
\end{tabular}


histopatológica permiten realizar un diagnóstico precoz y establecer un tratamiento oportuno, como la suspensión inmediata de la warfarina y la sustitución por heparinas, plasma fresco y vitamina $\mathrm{K}$.

\section{Referencias}

1. Daly AK, King BP. Pharmacogenetics of oral anticoagulants. Pharmacogenetics 2003; 13: 247-252.

2. Ansell J; Hirsh J; Hylek E; Jacobson A; Crowther M; Palareti G. Pharmacology and management of the vitamin K antagonists: American College of Chest Physicians Evidence-Based Clinical Practice Guidelines (8th Edition). Chest 2008 Jun; 133(6 Suppl): 160S-198S.

3. Flood EP, Redish MH, Bociek $\mathbf{S} \mathbf{J}$, et al. Thrombophlebitis migrans disseminata: Report of a case in which gangrene of a breast occurred. $N$ Y State J Med 1943; 43: 1121-1124.

4. Verhagen H. Local hemorrhage and necrosis ofthe skin and underlying tissues during anticoagulant therapy with dicumarol and dicumacyl. Acta Med Stand 1954; 148: 453 .
5. Warkentin TE, Sikov WM, Lillicrap DP. Multicentric warfarin-induced skin necrosis complicating heparininduced thrombocytopenia. Am J Hematol 1999 62: 44.

6. Kouris E, Kok E, Torres Z, Ball E, González F, Lugli M. Necrosis cutánea inducida por el uso de warfarina. Dermatol Venez 2005; 43: 24-26.

7. Cameron A, van Berkel W, Sixma J. Skin necrosis after three years of treatment with acenocoumarin. Ned Tijdschr Geneeskd 1974; 118: 505-507.

8. Becker CG. Oral anticoagulant therapy and skin necrosis: Speculations on pathogenesis. Adv Exp Med Biol 1987; 214: 217.

9. Lewandowski $\mathbf{K}$, Zawilska $\mathrm{K}$. Protein $\mathrm{C}$ concentrate in the treatment of warfarin-induced skin necrosis in the protein $\mathrm{C}$ deficiency. Thromb Haemost 1994; 71: 395.

10. Nazarian R, Van Cot E, Zembowicz A, Duncan L. Warfarin-induced skin necrosis. J Am Acad Dermatol 2009; 61: 325-32.

11. Reyes A, Raffo M, Masia G, Siegel $\mathbf{S}$, Gaete J, Vergara J et al. Necrosis cutánea por tratamiento anticoagulante oral. Caso clínico. Rev Chil Cir 2011; 63: 200-203.

12. Urbano-Ispizua A, Gaya A, Colado E, López M, Arrizabalaga B, Vicente V et al. Diagnóstico y tratamiento de la hemoglobinuria paroxística nocturna. Med Clin Barc 2011; 136: 121-127. 